\title{
Processed meat consumption and lung function: modification by antioxidants and smoking
}

\author{
Hitomi Okubo ${ }^{1,2}$, Seif O. Shaheen ${ }^{3}$, Georgia Ntani ${ }^{1}$, Karen A. Jameson', \\ Holly E. Syddall ${ }^{1}$, Avan Aihie Sayer ${ }^{1}$, Elaine M. Dennison ${ }^{1}$, Cyrus Cooper ${ }^{1}$, \\ Sian M. Robinson ${ }^{1}$ and the Hertfordshire Cohort Study Group
}

Affiliations: ${ }^{1}$ MRC Lifecourse Epidemiology Unit, University of Southampton, Southampton, and ${ }^{3}$ Centre for Primary Care and Public Health, Blizard Institute, Barts and The London School of Medicine and Dentistry, Queen Mary University of London, London, UK. ${ }^{2}$ Dept of Social and Preventive Epidemiology, Graduate School of Medicine, The University of Tokyo, Tokyo, Japan.

Correspondence: S.O. Shaheen, Centre for Primary Care and Public Health, Blizard Institute, Barts and The London School of Medicine and Dentistry, 58 Turner Street, London, E1 2AB, UK.

E-mail: s.shaheendamul.ac.uk

ABSTRACT Unhealthy dietary patterns are associated with poor lung function. It is not known whether this is due to low consumption of antioxidant-rich fruit and vegetables, or is a consequence of higher intakes of harmful dietary constituents, such as processed meat.

We examined the individual and combined associations of processed meat, fruit and vegetable consumption and dietary total antioxidant capacity (TAC) with lung function among 1551 males and 1391 females in the UK in the Hertfordshire Cohort Study. Diet was assessed using a food frequency questionnaire.

After controlling for confounders, processed meat consumption was negatively associated with forced expiratory volume in $1 \mathrm{~s}(\mathrm{FEV} 1)$, forced vital capacity (FVC) and FEV1/FVC ratio in males and females, while fruit and vegetable consumption and dietary TAC were positively associated with FEV1 and FVC, but not FEV1/FVC ratio. In males, the negative association between processed meat consumption and FEV1 was more marked in those who had low fruit and vegetable consumption ( $\mathrm{p}=0.035$ for interaction), and low dietary TAC ( $\mathrm{p}=0.025$ for interaction). The deficit in FEV1/FVC associated with processed meat consumption was larger in males who smoked ( $p=0.022$ for interaction).

Higher processed meat consumption is associated with poorer lung function, especially in males who have lower fruit and vegetable consumption or dietary TAC, and among current smokers.

@ERSpublications

Relative intake of foods that influence oxidant/antioxidant balance may be important for optimal lung function http://ow.ly/t6tgF

For editorial comments see page 943.

This article has supplementary material available from www.erj.ersjournals.com

Received: June 272013 | Accepted after revision: Oct 172013 | First published online: Oct 312013

Support statement: This study was supported by the Medical Research Council, UK. H. Okubo was supported in part by fellowship of the Astellas Foundation for Research on Metabolic Disorders, Japan and the Naito Memorial Grant for Research Abroad from the Naito Foundation, Japan.

Conflict of interest: Disclosures can be found alongside the online version of this article at www.erj.ersjournals.com

Copyright @ERS 2014 


\section{Introduction}

Healthy dietary patterns appear to have protective effects on lung function in older age [1-3]. For example, we have previously described a healthy "prudent" dietary pattern, characterised by high consumption of fruit, vegetables, oily fish and wholemeal cereals, but low consumption of white bread, added sugar, full-fat dairy products, chips and processed meat, that is associated with better lung function and reduced prevalence of chronic obstructive pulmonary disease (COPD) among older people in the UK [1]. The apparently beneficial effects of healthy dietary patterns on lung function have been largely attributed to the effects of antioxidants in fruit and vegetables. Epidemiological studies have reported positive associations of antioxidant-rich foods and nutrients, including vitamin $C$, vitamin $E, \beta$-carotene and flavonoids, with lung function [4-6]. Antioxidants are thought to play a protective role in the pathogenesis of lung impairment by scavenging free radicals and other oxygen species that cause cellular damage and inflammation [7-9].

However, one aspect of dietary patterns that may be overlooked is that they describe a balance of foods, and are characterised both by relatively high and low consumption of individual food items. Potentially one of the most important types of food that is less common in healthy diets is processed meat (such as bacon, ham, sausage and other cured meats). There is growing evidence that high consumption of processed meat is associated with poorer lung function and an increased risk of COPD, including exacerbations [10-13]. This could be due to its high nitrite content. Nitrites are added as preservatives, antimicrobial agents and colour fixatives, and generate reactive nitrogen species that can cause oxidative and nitrative damage to the lung [14]. Processed meat is also rich in advanced glycation end-products (AGEs) [15], which can increase oxidative stress and inflammation [16]. Thus, the size of the effect of processed meat consumption on lung function may also depend on other factors that influence pulmonary oxidant/antioxidant balance, such as dietary antioxidant intake and smoking. The protective effect of a healthy "prudent" dietary pattern on lung function could therefore reflect a favourable balance of protective antioxidants and harmful pro-oxidant foods in the diet. To our knowledge, the role of the balance of such foods in the diet has not been considered before.

In a large cohort of older males and females, we investigated associations between lung function and key foods (processed meat and fruit and vegetables) that may contribute to pulmonary oxidant/antioxidant balance. Our particular aim was to consider the nature of their individual and combined associations with lung function. In addition, since foods other than fruit and vegetables contribute to overall antioxidant status, we considered the role of the total antioxidant capacity (TAC) of the diet [17].

\section{Methods}

\section{The Hertfordshire Cohort Study}

Details of the Hertfordshire Cohort Study have been published elsewhere [18]. From 1911 to 1948, midwives recorded information on all infants born in the county of Hertfordshire, UK. In 1998, 3822 males and 3284 females (born 1931-1939) were traced. Permission to contact 3126 males (82\%) and 2973 females (91\%) was obtained from their general practitioner; 1684 males (54\%) and 1541 females (52\%) agreed to a home interview; and 1579 males (94\%) and 1418 females (92\%) attended a clinic for further investigations. In total, 1551 males and 1391 females completed spirometry tests. The study had ethical approval from the Bedfordshire and Hertfordshire local research ethics committee and the West Hertfordshire local research ethics committee. Written informed consent was obtained from all participants.

\section{Dietary assessment}

Diet over a 3-month period before the home interview was assessed using a food frequency questionnaire (FFQ) that was administered by a trained research nurse $[19,20]$. The FFQ included 129 foods and food groups. 10 predefined frequency responses were listed, ranging from "never" to " $\geqslant 6$ per day". Information on the frequency of consumption and quantities consumed of different types of alcoholic beverages was obtained separately. Energy intake from foods and alcoholic beverages was calculated by multiplying the frequency of consumption of a portion of each food item by its energy content, according to the UK national food composition database or manufacturers' composition data [21, 22].

For the analyses we grouped foods listed on the FFQ as follows. Processed meat: bacon and gammon, ham, corned beef, spam and luncheon meat, sausage, meat pies; fruit: fresh fruit (including citrus, apples, bananas and grapes), fruit juices, dried fruit, tinned and cooked fruit; vegetables: fresh and frozen vegetables (including cabbage, cauliflower, peas and root vegetables), salad vegetables, pulses, vegetarian foods and tinned vegetables. Weekly consumption (servings per week) of processed meat and fruit and vegetables were calculated as the sum of the individual frequencies of the foods within these groups.

Dietary TAC was estimated using published composition data where TAC was assessed using an oxygen radical absorbance capacity (ORAC) assay, which measured the degree of inhibition of 
peroxy-radical-induced oxidation in vitro $[23,24]$. ORAC was selected as one of the most widely used methods because of its biological relevance to antioxidant efficacy in vivo [23, 24]. A total dietary TAC score was calculated for each participant by multiplying the TAC values of each food/beverage by their reported frequency of consumption and then summing these values. A full description of the assignment of TAC values to each food item and calculation method is given in the online supplementary material.

\section{Lung function}

Lung function was measured using a Micro Spirometer (CareFusion UK, Gillingham, UK) in the seated position without noseclips. After at least one practice blow, three forced expiratory volume in $1 \mathrm{~s}$ (FEV1) and forced vital capacity (FVC) readings were recorded. The highest FEV1 and FVC values from satisfactory manoeuvres were used in the analyses; these did not necessarily come from the same blow. A bronchodilator was not given before spirometry was performed. For FEV1, $85.8 \%$ of males and $92.2 \%$ of females had a difference of $\leqslant 0.15 \mathrm{~L}$ between their two highest readings; for FVC, the corresponding figures were $80.4 \%$ and $88.6 \%$. However, we did not exclude those with a difference of $>0.15 \mathrm{~L}$ [25]. We calculated standardised residuals of lung function by using Global Lung Initiative 2012 regression equations (which are based on lung function data from lifelong nonsmokers) [26]. COPD was defined as FEV1/FVC ratio less than the lower limit of normal (i.e. the z-score was $<-1.645$ ) [26].

\section{Statistical analysis}

All statistical analyses were performed using Stata (version 12; StataCorp LP, College Station, TX, USA). Univariate and multiple linear regression analyses were used to examine the relationships between consumption of processed meat, fruit and vegetables, and dietary TAC with lung function outcomes; logistic regression was used to analyse COPD. For the regression analysis, we controlled for the effects of age and height, and the following potential confounders: smoking status (never, ex-, or current), pack-years smoked, exposure to tobacco smoke in the home, age at which the participant left education, social class, body fat mass, physical activity score, dietary supplement use, use of inhaled or oral steroids, use of paracetamol, alcohol consumption and energy intake. A detailed full description of the confounders is given in the online supplementary material. Tests for trend associations were based on continuously distributed variables and after adjustment for potential confounders.

We evaluated the combined associations of processed meat consumption and dietary antioxidants (i.e. fruit and vegetables and dietary TAC) on lung function, examining both their independent and interactive associations. We also stratified dietary associations by smoking status and tested for interactions. We analysed males and females separately. Where dietary variables were categorised into fifths (for tables) or thirds (for figures), we used cut-offs that were defined using the distributions for the whole population.

\section{Results}

The males and females studied were of similar social class, but the males were more likely to be current or ex-smokers, to smoke more heavily and to drink alcohol (table 1). The males were less likely than the females to be taking oral or inhaled steroids, paracetamol or dietary supplements. They had higher processed meat consumption and total energy intakes than females, but lower consumption of fruit and vegetables (all $\mathrm{p}<0.05)$. Dietary TAC was mainly derived from fruit $(35.8 \%)$, tea $(15.4 \%)$, vegetables (14.9\%), potatoes $(13.4 \%)$ and cereal products $(7.1 \%)$; dietary TAC did not differ between males and females. Males had a lower FEV1/FVC ratio and FEV1/FVC z-score and a higher FVC $\mathrm{z}$-score and prevalence of COPD (both $\mathrm{p}<0.001$ ).

\section{Individual associations of processed meat, fruit and vegetable consumption and dietary TAC with} lung function

After controlling for potential confounders, processed meat consumption was negatively associated with FEV1 in both sexes; this was especially marked in males (difference in FEV1 comparing top versus bottom fifth of consumption (95\% CI) $-170 \mathrm{~mL}(-250--80 \mathrm{~mL})$ ) (tables 2 and 3). In contrast, fruit and vegetable consumption and dietary TAC were positively associated with FEV1 in both males and females. The patterns of association with FVC were similar to those for FEV1. Processed meat consumption was negatively associated with $\mathrm{FEV} 1 / \mathrm{FVC}$ ratio, but there were no associations with fruit and vegetable consumption or dietary TAC in either males or females (tables 2 and 3). In males, processed meat consumption was positively associated with COPD ( $\mathrm{p}$-value for trend 0.013) (online supplementary table E1). Fruit and vegetable consumption and dietary TAC were not associated with COPD risk in either sex. The effect sizes for the associations between dietary exposures and lung function were compared for males and females, but they did not differ substantially ( $\mathrm{p}$-value for all interactions $>0.05$ ). 
TABLE 1 Subject characteristics of males and females who participated in the Hertfordshire Cohort Study, UK

\begin{tabular}{|c|c|c|}
\hline & Males & Females \\
\hline Subjects & 1551 & 1391 \\
\hline Age years & $65.7 \pm 2.9$ & $66.6 \pm 2.7$ \\
\hline Height $\mathrm{cm}$ & $174.2 \pm 6.4$ & $160.9 \pm 5.9$ \\
\hline Fat mass kg & $23.3(19.0-28.4)$ & $28.2(23.2-33.8)$ \\
\hline Habitual activity score & $61.0 \pm 15.3$ & $59.1 \pm 15.7$ \\
\hline \multicolumn{3}{|l|}{ Smoking status } \\
\hline Never-smoker & $507(32.7)$ & $854(61.4)$ \\
\hline Ex-smoker & $806(52.0)$ & $402(28.9)$ \\
\hline Current smoker & $238(15.3)$ & $134(9.6)$ \\
\hline Smoking pack-years & $23(11-40)$ & $15(5-29)$ \\
\hline Exposure to tobacco smoke in the home & $203(13.5)$ & $163(11.9)$ \\
\hline \multicolumn{3}{|l|}{ Age left full-time education years } \\
\hline$\leqslant 14$ & $302(19.5)$ & $241(17.3)$ \\
\hline$\geqslant 15$ & $1249(80.5)$ & $1150(82.7)$ \\
\hline \multicolumn{3}{|l|}{ Social class } \\
\hline I, II and IIINM & $611(40.6)$ & 583 (41.9) \\
\hline IIIM, IV and V & $893(59.4)$ & $807(58.1)$ \\
\hline Oral or inhaled steroids & $107(6.9)$ & $127(9.1)$ \\
\hline Paracetamol & $105(6.8)$ & $178(12.8)$ \\
\hline Dietary supplements & 712 (45.9) & 824 (59.2) \\
\hline Alcohol consumption units per week & $8.9(2.3-19.5)$ & $1.5(0.0-5.5)$ \\
\hline \multicolumn{3}{|l|}{ Dietary intake } \\
\hline Processed meat servings per week & $4.0(2.5-6.2)$ & $3.0(1.9-4.5)$ \\
\hline Fruit and vegetables servings per week & $42.6(31.1-56.3)$ & $47.1(35.5-60.4)$ \\
\hline TAC $\mu \mathrm{mol} \cdot \mathrm{day}^{-1}$ & $15915(12425-19640)$ & 15915 (12641-19387) \\
\hline Total energy intake $\mathrm{kcal} \cdot$ day $^{-1}$ & $2433(2093-2796)$ & $1973(1705-2272)$ \\
\hline \multicolumn{3}{|l|}{ Lung function } \\
\hline Maximum FEV1 L & $2.84 \pm 0.60$ & $1.98 \pm 0.41$ \\
\hline Maximum FVC L & $4.04 \pm 0.74$ & $2.71 \pm 0.50$ \\
\hline $\mathrm{FEV}_{1} / \mathrm{FVC}$ & $0.702 \pm 0.089$ & $0.732 \pm 0.079$ \\
\hline GLI 2012 FEV1 z-score ${ }^{\#}$ & $-0.74 \pm 0.03$ & $-0.77 \pm 0.03$ \\
\hline GLI 2012 FVC z-score ${ }^{\#}$ & $-0.28 \pm 0.03$ & $-0.42 \pm 0.03$ \\
\hline GLI 2012 FEV1/FVC z-score ${ }^{\#}$ & $-0.81 \pm 0.03$ & $-0.68 \pm 0.03$ \\
\hline Prevalence of COPD & $274(17.7)$ & $185(13.3)$ \\
\hline
\end{tabular}

Data are presented as $\mathrm{n}$, mean $\pm \mathrm{SD}$, median (interquartile range) or $\mathrm{n}(\%)$. I: professional; II: managerial and technical; IIINM: skilled nonmanual; IIIM: skilled manual; IV: partly skilled manual; V: unskilled manual; TAC: total antioxidant capacity; FEV1: forced expiratory volume in $1 \mathrm{~s}$; FVC: forced vital capacity; GLI: Global Lungs Initiative; COPD: chronic obstructive pulmonary disease. ${ }^{\#}$ : calculated using the GLI 2012 regression equations (which are based on lung function data from lifelong nonsmokers) [26].

Combined associations of processed meat consumption and dietary antioxidants with lung function Processed meat consumption was weakly correlated with fruit and vegetable consumption (Spearman correlation coefficients $r=-0.06, p=0.01$ for males and $r=-0.07, p=0.01$ for females), but not dietary TAC $(\mathrm{r}=-0.05, \mathrm{p}=0.05$ for males and $\mathrm{r}=-0.03, \mathrm{p}=0.21$ for females). We therefore investigated the independent associations between lung function and processed meat, fruit and vegetable consumption and dietary TAC by mutual adjustment in multivariate models. The negative association between processed meat consumption and FEV1 was independent of fruit and vegetable consumption and dietary TAC in males ( $\mathrm{p}$-value for trend both $<0.001$ ) and females ( $\mathrm{p}$-value for trend 0.013 and 0.014 , respectively). Similarly, associations between processed meat consumption and FVC and FEV1/FVC ratio in males and females, and COPD in males, were not confounded by fruit and vegetable consumption or dietary TAC (data not shown). In contrast, the positive associations of fruit and vegetable consumption and dietary TAC with FEV1 and FVC remained after adjustment for processed meat consumption in females, but these associations disappeared in males.

We then examined whether the association between processed meat consumption and FEV1 was modified by fruit and vegetable consumption and dietary TAC (fig. 1). In males, the association between processed 


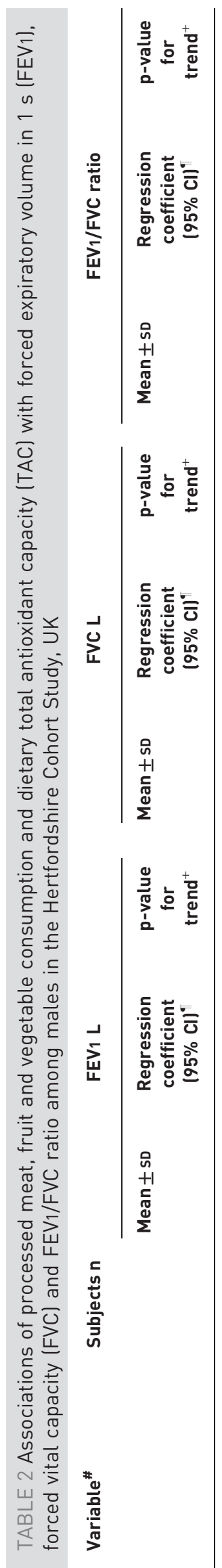

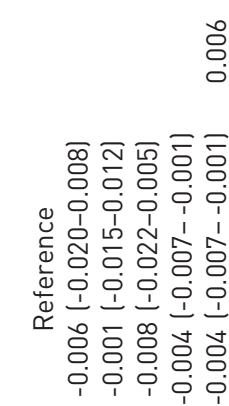

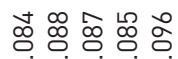

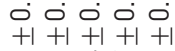

옹옹ㅎㅇ중

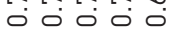

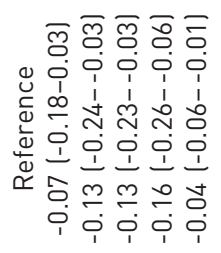

ํㅗㅊํำ

0000

$+1+1+1+1+1$

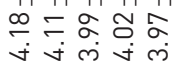

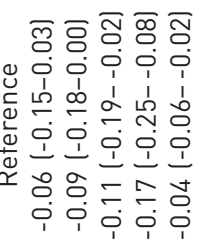

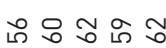

+1
+1

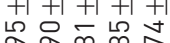

กั่

농요 좃

চ.

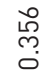

흉ㅇㅇㅇ

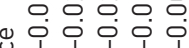

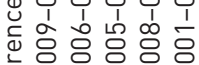

ब范

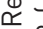

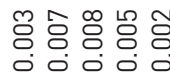

mo

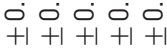

잉응ㅎㅇㅇ

ऽ。

잉

흐몽

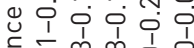

는

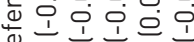

드으으응

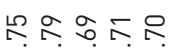

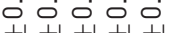

$+1+1+1+1+1$

m

ఫ̊

뜨종

$\begin{array}{lllll}0 & 0 & 0 & 0 \\ 0 & 1 & 0 & 0 \\ 0 & 1 & 1 & 1 & 1 \\ 0 & 0 & 0 & 0 & 0 \\ 0 & 0 & 0 & 0 \\ 0 & 0 & 0 & 0 & 0 \\ 0\end{array}$

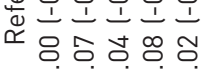

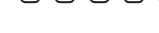

농ํำ

$9+1+1+1+1$
$0+1+1+1$

요 요 잉

ก $\sim \sim \sim \sim$

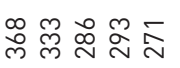

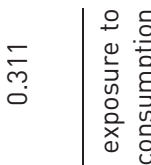

귱쥬융

$\begin{array}{llllll}0 & 1 & 0 & 0 & 0 & 0 \\ 0 & 1 & 1 & 1\end{array}$

๘

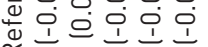

응형ㅎㅇ

$0 \circ 0$ O

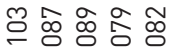

0.000

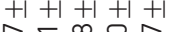

엉ㅎㅇㅇ으옹

O० O. O.

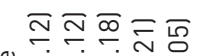

Ð

๘

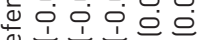

종응ㅇㅇㅇ

ะำำ

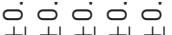

$+1+1+1+1+1$

응유늠

m过过的

.

m흠ㅁㅇㅁ

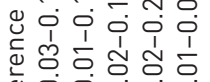

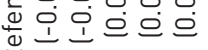

늠드응

००

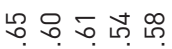

000

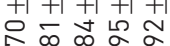

ก ก

m $\frac{m}{m} \stackrel{\infty}{\sim} \stackrel{\sim}{\sim} \underset{\sim}{~}$

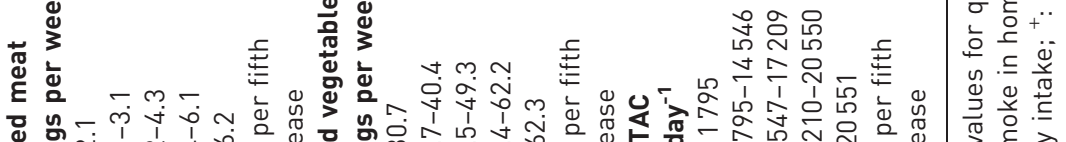

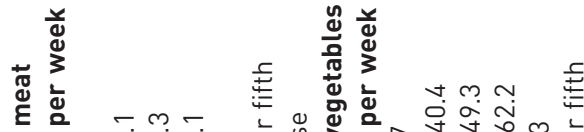

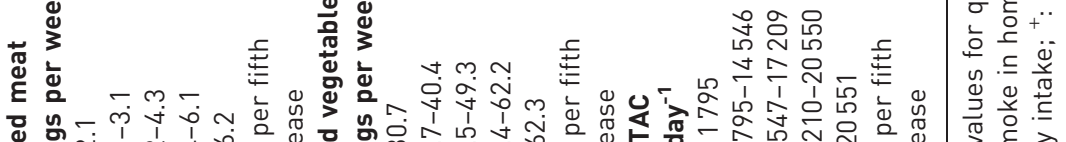

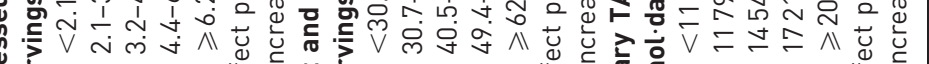
岁厅 


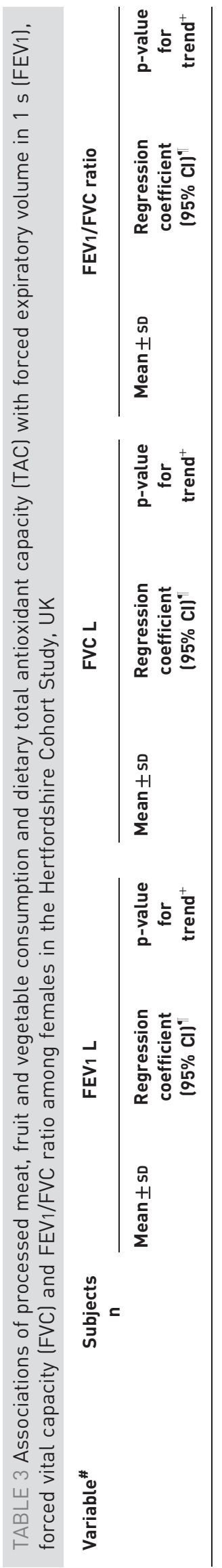

ఫ্

ले

:

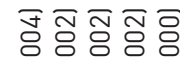

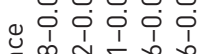

๘

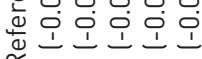

동응으웡ㅇ

i cio i i

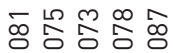

0.000000

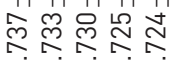

o. o.

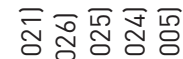

\& 0 i 10 i

๘응용ㅎㅇ

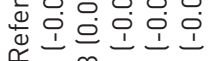

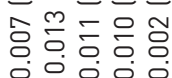

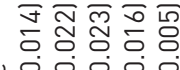

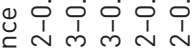

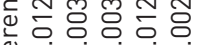

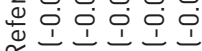

훙응흥훙

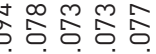

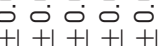

쵸요요

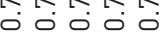

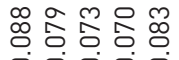

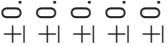

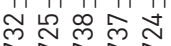

。ㅇㅇㅇㅇㅇㅇㅇㅇ

ס̊.

₹ㅎㅎㅎㅎㅎㅁ

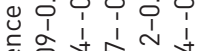

a

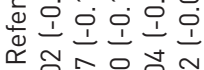

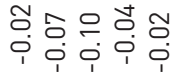

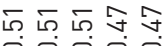

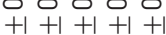

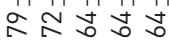

ivivi

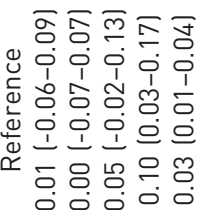

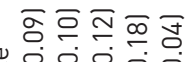

\&

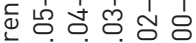

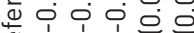

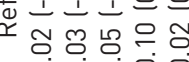

0.000

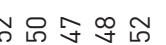

00000

$+1+1+1+1+1$

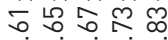

섯ำ

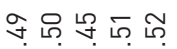

0.00

$+1+1+1+1+1$

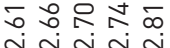

$\stackrel{2}{\circ}$

চ̊

호.

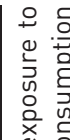

家

旁苧

苋

密

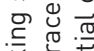

है

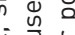

莒司 흥

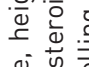

常苋

这妾

旁管.

$\because{ }^{\circ}$

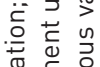

흥 응

䆑

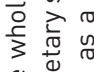

这

कृष

है un

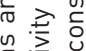

资忞过

考 $\frac{\pi}{\pi} \frac{\pi}{\frac{\pi}{0}}$

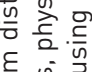

즣ㅎㅎㅎ

\& 1010

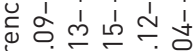

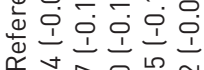

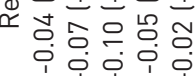

흫ㅎㅎ

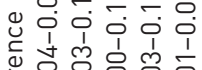

๘

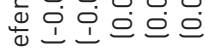

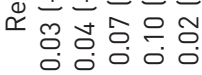

₹워워

$\begin{array}{lll}2 & 0 \\ 0 & 0 & 0\end{array}$

ㅇํํ의

寸ㄱำ

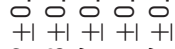

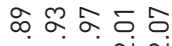

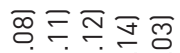

\& ${ }_{1}$ i

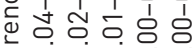

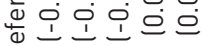

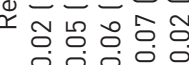

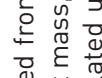

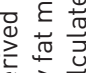

항 증 정

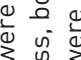

3 每

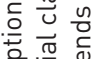

궘의 웡 ᅯ

0 .

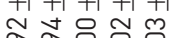

‥ n i

承

స્心

$\stackrel{\infty}{m} \stackrel{m}{m} \stackrel{\infty}{\sim} \stackrel{\sim}{N} \underset{m}{\sim}$

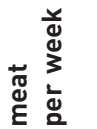

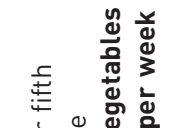

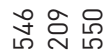

츤

守

茯

在管

要 范

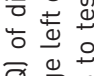

으 하

造焉

음오

흘

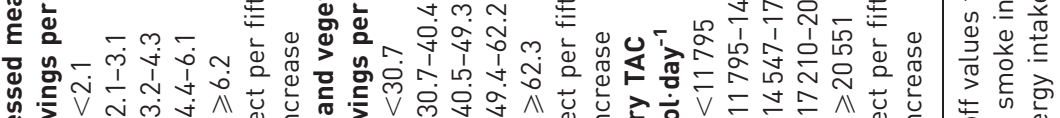

पे के

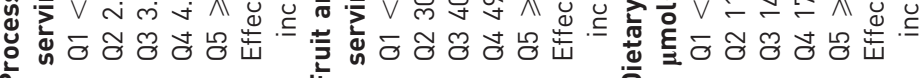

ㅇํㅇ

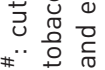



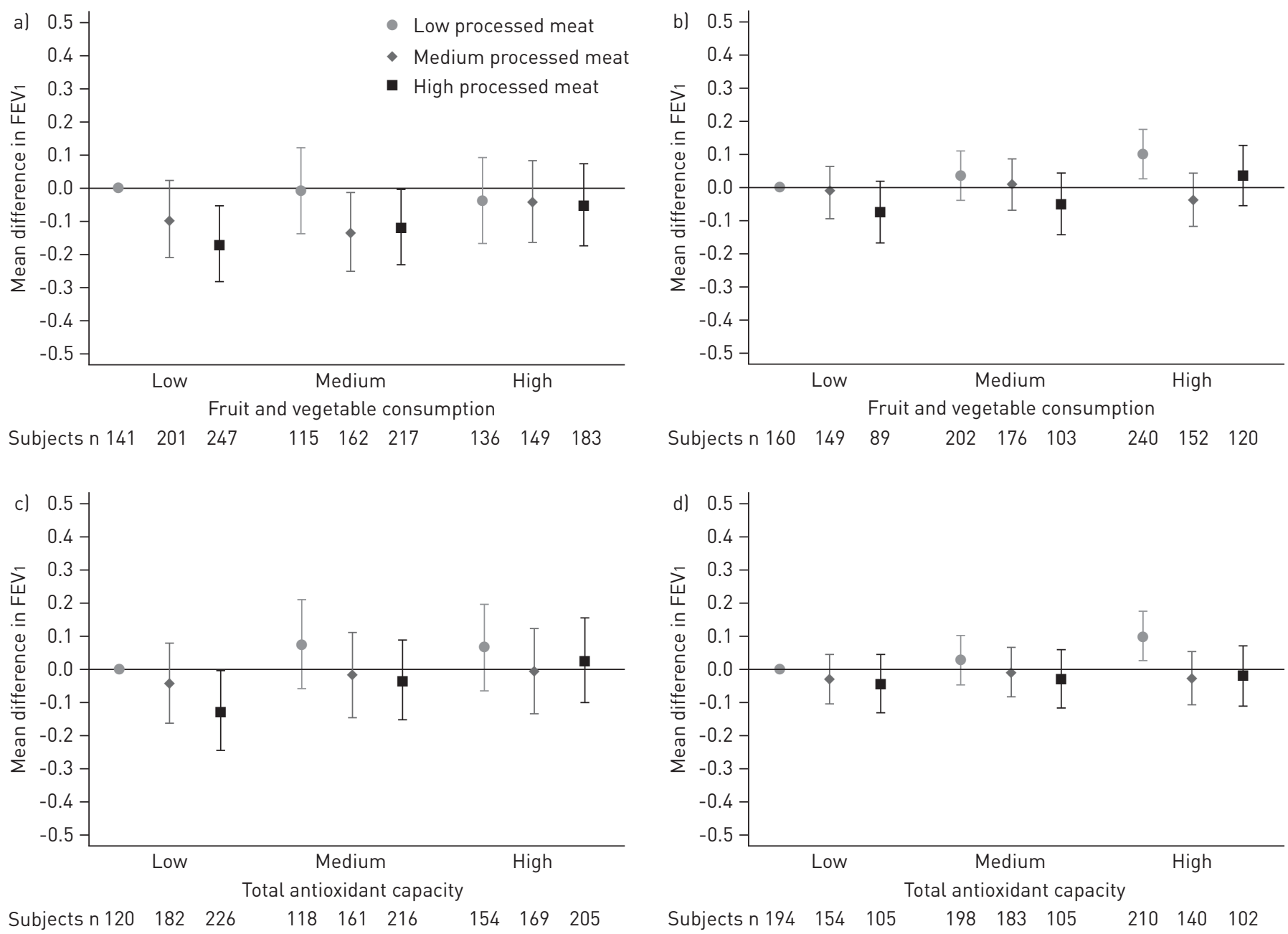

FIGURE 1 Interactions between processed meat consumption and fruit and vegetable consumption in a) males and b) females, and dietary total antioxidant capacity (TAC) in c) males and d) females, on forced expiratory volume in $1 \mathrm{~s}$ (FEV1). Dietary variables were stratified by tertiles (processed meat: low $<2.6$ servings per week, medium 2.6-4.8 servings per week and high $\geqslant 4.9$ servings per week; fruit and vegetables: low <37.5 servings per week, medium $37.5-53.1$ servings per week and high $\geqslant 53.2$ servings per week; and dietary TAC: low $<13700 \mu \mathrm{mol} \cdot$ day $^{-1}$, medium: $13700-18245 \mu$ mol $\cdot$ day $^{-1}$ and high $\geqslant 18246 \mu \mathrm{mol} \cdot$ day $\left.^{-1}\right)$. Values are multivariate-adjusted regression coefficients for the difference in mean FEV1 (95\% CI) compared to subjects with the lowest consumption of both processed meat and dietary antioxidants.

meat consumption and FEV1 was more marked in those who had lower fruit and vegetable consumption (p-value for interaction 0.035) (fig. 1a) and lower dietary TAC (p-value for interaction 0.025) (fig. 1c). In females, the association between processed meat consumption and FEV1 did not differ according to fruit and vegetable consumption ( $\mathrm{p}$-value for interaction 0.633 ) or dietary TAC ( $\mathrm{p}$-value for interaction 0.412 ). There was no evidence of effect modification in relation to FVC (data not shown), FEV1/FVC ratio (online supplementary fig. E1) or COPD (data not shown) in either males or females.

\section{Interactions between dietary consumption and smoking status on lung function}

In both males and females there was some evidence that negative associations of processed meat consumption, and positive associations of fruit and vegetable consumption and dietary TAC, with FEV1 were more marked in smokers, although the tests for interaction did not achieve conventional statistical significance (i.e. $\mathrm{p}<0.05$ ) (fig. 2). The patterns of association with FVC and COPD were similar to those for FEV1 (data not shown). However, for FEV1/FVC ratio we found that the negative association with processed meat consumption among males was stronger in current smokers ( $p$-value for interaction 0.022) (online supplementary fig. E2), but no clear differences in the associations with fruit and vegetable consumption or dietary TAC according to smoking status were observed ( $\mathrm{p}$-values for interaction 0.108 and 0.595 , respectively). In females, there were no clear differences in the associations between dietary exposures and FEV1/FVC ratio according to smoking status. 

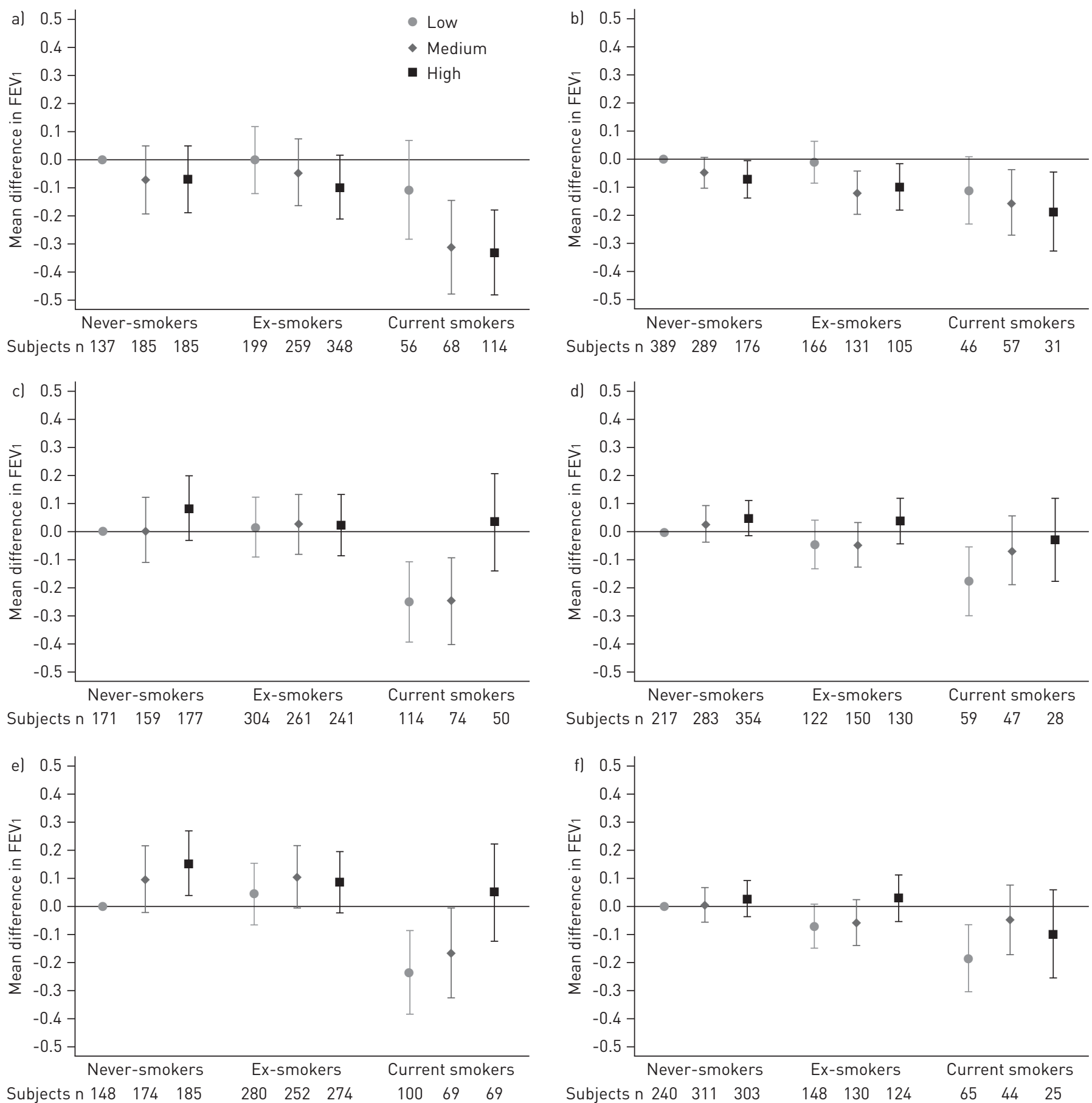

FIGURE 2 Interactions between processed meat consumption for a) males and b) females, fruit and vegetable consumption for c) males and d) females and dietary total antioxidant capacity (TAC) for e) males and f) females and smoking status on forced expiratory volume in $1 \mathrm{~s}$ (FEV1). Dietary variables were stratified by tertiles (processed meat: low $<2.6$ servings per week, medium $2.6-4.8$ servings per week and high $\geqslant 4.9$ servings per week; fruit and vegetables: low $<37.5$ servings per week, medium $37.5-53.1$ servings per week and high $\geqslant 53.2$ servings per week; and dietary TAC: low $<13700 \mu$ mol $\cdot$ day ${ }^{-1}$, medium: $13700-18245 \mu \mathrm{mol} \cdot$ day $^{-1}$ and high $\geqslant 18246 \mu \mathrm{mol} \cdot$ day $\left.^{-1}\right)$. Values are multivariate-adjusted regression coefficients for the difference in mean FEV1 $(95 \%$ CI) compared to never-smokers with the lowest consumption of each dietary component.

\section{Discussion}

\section{Main findings}

The main findings of this study were that higher processed meat consumption was associated with poorer lung function in older males and females, and that in males the negative association with FEV1 was greatest in those who also had lower fruit and vegetable consumption and low dietary TAC. These interactions 
suggest that the association between a healthier dietary pattern and lung function, as observed previously in this cohort [1] and in other populations [2,3], might not simply reflect the intake of antioxidants, but rather the relative intakes of protective and harmful constituents in the diet that influence pulmonary oxidant/antioxidant balance. To our knowledge, the effects of the balance of foods in the diet in relation to lung function have not been described before.

\section{Association of processed meat consumption with lung function}

Our findings of poorer lung function among males and females who have a high consumption of processed meat are consistent with a growing number of epidemiological studies of lung function and COPD from the USA [10-12] and Europe [13]. In keeping with findings from the cross-sectional study of the National Health and Nutrition Examination Survey (NHANES) III [10], we confirmed a negative association of processed meat consumption with FEV1 and FEV1/FVC ratio and a positive association with COPD, defined spirometrically. In contrast, we also found a negative association with FVC. A further difference between studies is our observation of an interaction between smoking and processed meat consumption in relation to FEV1/FVC ratio in males, which was not seen in NHANES III. However, our study suggests that there may be sex-specific effects of diet on lung function that were not examined previously [10].

A number of potential mechanisms have been suggested to link processed meat consumption with poorer lung function. A key component of processed meat is its high nitrite content [10-13]. Nitrites are prooxidants and can generate strong oxidising reactive nitrogen species, such as peroxynitrite [14], which can produce lung damage and contribute to airway inflammation. Tobacco smoke is another source of nitrites as well as oxidants; hence the interaction between processed meat consumption and smoking on lung function in males is biologically plausible. In addition, cured/processed meats are a rich source of AGEs [15]. AGEs contribute to increased oxidative stress and inflammation through binding with their cell surface receptor, which activates nuclear factor (NF)- $\mathrm{\kappa B}[16,27]$. Thus high consumption of foods rich in AGEs could plausibly increase lung inflammation and hence reduce lung function. Given the likely prooxidant effects of nitrites and AGEs in processed meat, it may be unsurprising that the association between processed meat consumption and FEV1 was modified by fruit and vegetable consumption and dietary TAC, at least in males. The significant trends in the associations of processed meat consumption with lung function, suggestive of a "dose response", the magnitude of the association with FEV1, and the plausible interactions with smoking and dietary antioxidants in males would support a causal interpretation.

\section{Association of dietary antioxidants with lung function}

Consistent with the growing recognition of potential beneficial effects of foods rich in antioxidants on lung function [6], we found that fruit and vegetable consumption and dietary TAC were positively associated with FEV1 and FVC. Comparable associations with dietary TAC have also been described in an Italian study where dietary TAC was positively associated with FEV1 and FVC, although these effects were seen only in females [28]. Dietary TAC reflects all antioxidants in the diet and even takes into account their synergistic effects $[17,23,24]$; hence dietary TAC is expected to be more useful than a single-food or single-nutrient approach to examine relationships between antioxidants and health outcomes. However, in our study the effects of dietary TAC on lung function were not markedly different from those of fruit and vegetables (tables 2 and 3). One possible explanation is that our estimation of dietary TAC was inaccurate. This may partly be because of a lack of TAC values in the database we used to assign to the foods on the FFQ (coverage rate $44.8 \%$ ), but also because the database, developed in the USA, may not be appropriate to estimate TAC in foods eaten in the UK, where growing conditions and cooking methods differ. To our knowledge dietary TAC using the ORAC assay has not been described in other UK cohort studies, and further data, using other estimates of dietary TAC, are needed to understand its importance for health.

\section{Sex-specific effects of diet on lung function}

Although higher processed meat consumption was associated with reduced lung function in both males and females, the effect modification by dietary antioxidants and smoking was observed only in males. It is not clear why the effect modification of diet differs between males and females, although comparable sex differences in oxidative stress have been described in another study [29].

\section{Study limitations}

Our study has a number of limitations. First, the study is cross-sectional, which limits causal inference. However, "reverse causation" does not seem a likely explanation for the main findings as we cannot see why individuals developing worse lung function would choose to eat more processed meat or fewer fruit and vegetables. Secondly, while we defined COPD spirometrically, which is the gold-standard approach [30] and avoids potential problems of bias which might arise with self-reported COPD, we did not measure postbronchodilator lung function. This raises the possibility that a small minority of individuals classified as 
having COPD by our spirometric definition may have had asthma. However, such misclassification of phenotype would seem less likely in males, in whom the association between processed meat consumption and FEV1/FVC was modified by smoking, the main risk factor for COPD. Thirdly, dietary information was collected using an administered FFQ. There are concerns that participants can over-report intake in response to FFQs, although their ability to describe types of diets and patterns of food consumption is well established [20, 22]. However, as measurement error and misclassification of exposure is likely to be random with respect to the study outcomes, this would be expected to attenuate associations; therefore, we do not think that misreporting on the FFQ could explain the associations we describe. Finally, subjects with high fruit and vegetable consumption and dietary TAC or with low processed meat consumption may be more health conscious and have other healthy behaviours, which could potentially confound associations with lung function. While we controlled for a large number of potential confounders, including detailed measures of smoking status, we cannot rule out unmeasured or residual confounding (such as the other environmental sources of oxidants/antioxidants, air pollution and occupational exposures) in an observational study of this kind.

\section{Conclusion}

Processed meat consumption was negatively associated with lung function in both males and females. This association was stronger among males with low fruit and vegetable consumption, low dietary TAC and among current smokers. Smoking cessation remains the most important public health message to prevent reduced lung function. However, the present findings suggest that the relative consumption of foods that influence pulmonary oxidant/antioxidant balance may also be important for optimising lung function, particularly in smokers. While longitudinal data are needed, these findings provide further evidence to suggest that current dietary guidelines to promote "healthier" patterns of eating could play a protective role in slowing lung function decline and preventing COPD in older age.

\section{Acknowledgements}

We thank all the participants of the Hertfordshire Cohort Study (HCS) and the HCS research staff.

\section{References}

1 Shaheen SO, Jameson KA, Syddall HE, et al. The relationship of dietary patterns with adult lung function and COPD. Eur Respir J 2010; 36: 277-284.

2 Varraso R, Fung TT, Hu FB, et al. Prospective study of dietary patterns and chronic obstructive pulmonary disease among US men. Thorax 2007; 62: 786-791.

3 Varraso R, Fung TT, Barr RG, et al. Prospective study of dietary patterns and chronic obstructive pulmonary disease among US women. Am J Clin Nutr 2007; 86: 488-495.

Romieu I, Trenga C. Diet and obstructive lung diseases. Epidemiol Rev 2001; 23: 268-287.

5 Carey IM, Strachan DP, Cook DG. Effects of changes in fresh fruit consumption on ventilatory function in healthy British adults. Am J Respir Crit Care Med 1998; 158: 728-733.

6 Schünemann HJ, Freudenheim JL, Grant BJ. Epidemiologic evidence linking antioxidant vitamins to pulmonary function and airway obstruction. Epidemiol Rev 2001; 23: 248-267.

7 Kelly FJ. Vitamins and respiratory disease: antioxidant micronutrients in pulmonary health and disease. Proc Nutr Soc 2005; 64: 510-526.

8 Mak JC. Pathogenesis of COPD. Part II. Oxidative-antioxidative imbalance. Int J Tuberc Lung Dis 2008; 12: 368-374.

9 Rahman I, MacNee W. Oxidant/antioxidant imbalance in smokers and chronic obstructive pulmonary disease. Thorax 1996; 51: 348-350.

10 Jiang R, Paik DC, Hankinson JL, et al. Cured meat consumption, lung function, and chronic obstructive pulmonary disease among United States adults. Am J Respir Crit Care Med 2007; 175: 798-804.

11 Jiang R, Camargo CA Jr, Varraso R, et al. Consumption of cured meats and prospective risk of chronic obstructive pulmonary disease in women. Am J Clin Nutr 2008; 87: 1002-1008.

12 Varraso R, Jiang R, Barr RG, et al. Prospective study of cured meats consumption and risk of chronic obstructive pulmonary disease in men. Am J Epidemiol 2007; 166: 1438-1445.

13 de Batlle J, Mendez M, Romieu I, et al. Cured meat consumption increases risk of readmission in COPD patients. Eur Respir J 2012; 40: 555-560.

14 Folkerts G, Kloek J, Muijsers RB, et al. Reactive nitrogen and oxygen species in airway inflammation. Eur J Pharmacol 2001; 429: 251-262.

15 Uribarri J, Woodruff S, Goodman S, et al. Advanced glycation end products in foods and a practical guide to their reduction in the diet. J Am Diet Assoc 2010; 110: 911-916.

16 Uribarri J, Cai W, Peppa M, et al. Circulating glycotoxins and dietary advanced glycation endproducts: two links to inflammatory response, oxidative stress, and aging. J Gerontol A Biol Sci Med Sci 2007; 62: 427-433.

17 Serafini M, Del Rio D. Understanding the association between dietary antioxidants, redox status and disease: is the Total Antioxidant Capacity the right tool? Redox Rep 2004; 9: 145-152.

18 Syddall HE, Aihie Sayer A, Dennison EM, et al. Cohort profile: the Hertfordshire cohort study. Int J Epidemiol 2005; 34: 1234-1242.

19 Bingham SA, Gill C, Welch A, et al. Comparison of dietary assessment methods in nutritional epidemiology: weighed records v. $24 \mathrm{~h}$ recalls, food-frequency questionnaires and estimated-diet records. Br J Nutr 1994; 72: 619-643. 
Robinson S, Syddall H, Jameson K, et al. Current patterns of diet in community-dwelling older men and women: results from the Hertfordshire Cohort Study. Age Ageing 2009; 38: 594-599.

21 Holland B, Welch AA, Unwin ID, et al. McCance and Widdowson's The Composition of Foods. 5th Edn. Cambridge, Royal Society of Chemistry, 1991, and supplements to this edition.

22 Robinson SM, Jameson KA, Batelaan SF, et al. Diet and its relationship with grip strength in community-dwelling older men and women: the Hertfordshire cohort study. J Am Geriatr Soc 2008; 56: 84-90.

23 Wu X, Beecher GR, Holden JM, et al. Lipophilic and hydrophilic antioxidant capacities of common foods in the United States. J Agric Food Chem 2004; 52: 4026-4037.

24 United States Department of Agriculture Agricultural Research Service. USDA Database for the Oxygen Radical Absorbance Capacity (ORAC) of Selected Foods, Release 2. Beltsville, Agricultural Research Service, 2010.

25 Miller MR, Hankinson J, Brusasco V, et al. Standardisation of spirometry. Eur Respir J 2005; 26: 319-338.

26 Quanjer PH, Stanojevic S, Cole TJ, et al. Multi-ethnic reference values for spirometry for the 3-95-yr age range: the global lung function 2012 equations. Eur Respir J 2012; 40: 1324-1343.

27 Semba RD, Nicklett EJ, Ferrucci L. Does accumulation of advanced glycation end products contribute to the aging phenotype? J Gerontol A Biol Sci Med Sci 2010; 65: 963-975.

28 di Giuseppe R, Arcari A, Serafini M, et al. Total dietary antioxidant capacity and lung function in an Italian population: a favorable role in premenopausal/never smoker women. Eur J Clin Nutr 2012; 66: 61-68.

29 Ochs-Balcom HM, Grant BJ, Muti P, et al. Oxidative stress and pulmonary function in the general population. Am $J$ Epidemiol 2005; 162: 1137-1145.

30 Celli BR, MacNee W, Agusti A, et al. Standards for the diagnosis and treatment of patients with COPD: a summary of the ATS/ERS position paper. Eur Respir J 2004; 23: 932-946. 